\title{
15
}

\section{Levels of analysis of the Vestibulo-Ocular Reflex: a post-modern approach}

\section{Laurence R. Harris, Karl Beykirch and Michael Fetter}

\subsection{Introduction}

This chapter examines some of the ways in which a basic motor act, the vestibuloocular reflex, can be considered. It takes its inspiration in part from Dr Ian Howard. Ian's functional approach with his emphasis on explanation-by-demonstration, has been hugely influential on the first author.

Which frame of reference is used by the reflexes that tend to maintain gaze during head movements is a question to which the answer has tended to be unnecessarily constrained by the anatomically rigid frame of the vestibular end organ embedded in the skull. Neural processes have no such constraint and indeed the frame they use cannot be based in the head since it is not possible to code head movement in a head-based co-ordinate system (Harris, 1997; Harris, Zikovitz and Kopinska, 1998). Here we model the eye movements evoked while rotating around various axes, as the output of a system consisting of three channels. The orientations of the channels needed to best model the eye movements evoked by rotation before and after an adaptation procedure have been determined. One was found close to the roll direction with the other two roughly in Listing's plane, and approximately equidistant from all three canal pairs.

\subsubsection{Channel theory}

The nervous system processes information. Information can be most efficiently processed if it is handled by a dedicated system or channel that carries information about only one thing. That way the decoder that receives the output can make assumptions about the significance of activity in that channel. However, even for a dedicated channel, some of that activity will not be relevant to the information 
the system is transmitting, even though it might be potentially useful and interesting information about something else. If the information within the channel can be carried as a difference between multiple sub- channels that are all equally affected by at least some sources of noise, then correlated noise is cancelled out. This is channel theory. The idea of channels in neural systems emerged from communication theory (Shannon and Weaver, 1949), see (Regan, 2000) and has since had a long and useful history in modelling neural information processing (Campbell and Tegeder, 1991; Harris, 1997). It is closely connected to the modular approach to modelling the brain (e.g., Zeki, 2001) and both channel and modules are the descendants of Müller's Principle of Specific Nerve Energies (Müller, 1840). Müller's principle states that modality of information is coded by which nerves or brain areas are active, leaving the decoder to specialize in decoding an already-known parameter.

Channels have been explicitly used to model many aspects of sensory information processing (Blum, 1991) but not overtly to describe "lower-level" information processing such as are involved in the essentially unconscious processes underlying so-called reflex eye movement control. Here we apply the concept of channels to modelling the coding the pattern of eye movements evoked by passive head rotation in the dark.

\subsubsection{Compensating for instability}

As we move around the world the movement threatens the precarious stability of our retinal image and our posture. Various compensatory responses allow us to minimize perceptual and physical instability and thus help us to continue to function as well as possible during the movement. Potential threats to ocular stability stimulate visual, vestibular and other proprioceptive systems which together, in the context of the task in hand, drive compensatory movements of the eye, head and body that tend to neutralize the threat. Each of the contributing systems, defined in terms of its sensory input and motor output, is capable of operating on its own and each system has traditionally been investigated alone with the implication that each can be viewed as an essentially independent sub-system.

The vestibulo-ocular reflex (VOR) in such a view, is the sub-system that measures head movement with the vestibular organs and generates a compensatory oculomotor response. The VOR has been regarded as a classic example of a simple reflex (Lorente de Nó, 1933; Szentágothai, 1950): the head moves to the left, the eyes move reflexively to the right thus cancelling the retinal disturbance that would otherwise have occurred. More recently, an amazing flexibility has become apparent in the VOR system and it has come to be regarded as a context-dependent motor response with an extraordinary adaptive ability. The VOR is able to adjust to a remarkable array of demands that are placed on it by virtue of the position of the two eyes in the head and the geometry of their relationship to objects in the outside world and it can be recalibrated in response to both long- and short-term demands of the environment, especially visual demands. 


\subsubsection{A post-modern approach}

Considering the vestibulo-ocular reflex as dependent on the ongoing context reflects the post-modern movement that, originating in architecture, now pervades many aspects of knowledge. Postmodernism rejects the modernist approach which celebrated the autonomous individual: a VOR capable of working perfectly in glorious isolation. In a post-modern world actions and thoughts can only be interpreted as part of the total environment in which they occur.

Early models of the VOR were based on the three-neurone arc concept (Lorente de Nó, 1933) that implied that the horizontal VOR (lateral eye movement evoked in response to yaw head rotation, see Fig. 1) was processed as an independent mechanism within the VOR system. Since yaw movement stimulates primarily the horizontal semicircular canal pair (Curthoys, Markham and Blanks, 1975), this approach implied that other canal pairs also had their own independent connections to the appropriate eye muscles. The idea of a set of three sub-systems defined by the semicircular canals within the skull underlying the generation of the VOR around any axis received support from the spatial tuning of sensory responses in various parts of the brain known to be involved in the neural interpretation of the VOR (Leonard, Simpson and Graf, 1988; Simpson, Graf and Leonard, 1981; Graf, Simpson and Leonard, 1988; Oyster, Takahashi and Collewijn, 1972). Furthermore the direction of pull of the oculomotor muscles themselves also seem to be arranged in planes roughly aligning with the orientation of the semicircular canal planes (Graf and Simpson, 1981).

As the rather sophisticated properties of the VOR emerged, regarding its neural substrate as simple, independent connections between the canals and the oculomotor muscles became less and less tenable. Amongst the VOR's features are the flexibility of its gain (the speed of the eye movement associated with a given speed of head movement) (Malcolm and Jones, 1970) and direction (Gonshor and Jones, 1976) in response to changes in visual demands (see Berthoz and Melvill Jones, 1985 for a review). Even the coupling between canal stimulation and which muscles are activated is flexible. The changes int he VOR in response to changes in the coupling required during natural changes in the course of some animal's lives (Graf and Baker, 1983; 1990) or imposed in the laboratory (Peng, Baker and Peterson, 1994) have shown how vestibularly transduced movement around a given axis can be coupled to eye movements around various axes depending on circumstances. The VOR also depends on the eye's instantaneous position in the orbit (Fetter, Hain and Zee, 1986; Fetter, Misslisch, Sievering and Tweed, 1995; Misslisch, Tweed, Fetter, Dichgans and Vilis, 1997; Misslisch and Hess, 2000), vergence (Mok, Ro, Cadera, Crawford and Vilis, 1992), the distance of regard (Biguer and Prablanc, 1981; Paige, Telford, Seidman and Barnes, 1998), and the orientation of the rotation axis relative to the head and relative to gravity (Hess and Angelaki, 1997). Although the VOR has been thought of as a purely angular phenomenon, nearly all rotations of the head cause a physical translation of the eyes because the centre of rotation of the eyes are not on the axis of most head rotations. The VOR is sensitive to this (Biguer and Prablanc, 1981; Viirre and 
Demer, 1995; Viirre, Milner, Tweed and Vilis, 1986) and is even responsive to extending the distance between the eyes and the axis of head rotation (Snyder and King, 1992).

Thus we have a post-modern reflex to consider: one that is sensitive to its context. We show that a simple three channel model can form a core onto which this flexible family of modifications can be appended.

\subsection{The three-dimensional performance of the vestibulo-ocular reflex}

We measured the VOR evoked in response to passive whole-body rotation about various axes in the dark to quantify its performance, especially its alignment with the stimulating axis. Deviations have been reported around pitch, roll and yaw axes (Biguer and Prablanc, 1981; Viirre and Demer, 1995; Viirre, Milner, Tweed and Vilis, 1986) but have been less well investigated in intermediate axes (but see Solomon, Straumann and Zee, 1997). We then altered the response around one axis and looked again at the variation of response amongst axes. Our aim was to test a channel-based model inspired by the success of channel-based models in describing other aspects of sensory processing. A detailed description of the methods can be found on the enclosed CD ROM and in Harris, Beykirch and Fetter, (in press).

The axes we used are shown in Fig. 15.1 and corresponded to the cardinal axes (roll, pitch and yaw) and are half way in between. The axes between roll and yaw we refer to as unicorn (forward tipping) and oryx (backwards tipping). The axes between roll and yaw correspond approximately to the planes of the vertical semicircular pairs: right anterior-left posterior (RALP) and left anteriorright posterior (LARP). The axes between the yaw and pitch we refer to as horns but only the left horn was available in the equipment's configuration. We used sum-of-sines stimuli, comprising four frequencies from 0.032 to $0.258 \mathrm{~Hz}$ with an amplitude of $\pm 20^{\circ}$ for each component. The sum of sines stimulus ensured that the stimulus was unpredictable. Three dimensional eye position signals were recorded using a coil system.

\subsubsection{VOR evoked by rotation about axes in the fronto-parallel plane}

When rotation was about axes in the fronto-parallel plane (yaw, pitch and left horn axes), compensatory eye movements were evoked that accurately aligned with the stimulating axis. The mean deviation from accurate alignment was only $2.0 \pm 0.4^{\mathrm{O}}$ with the largest deviation being for rotation about the left horn axis $\left(4.6^{\circ}\right)$. The exact values are given in table 1 on the enclosed CD. 


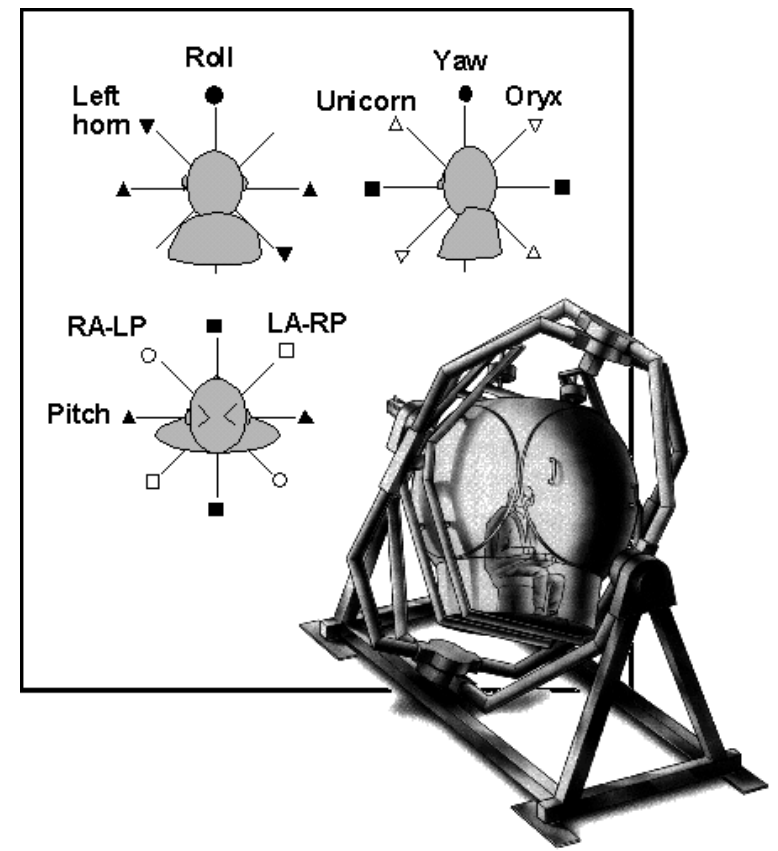

FIGURE 15.1. The axes used in this study seen in above, behind and the side views. The orientation of the vertical canals are shown diagrammatically in the above view. The insert is a picture of the Tübingen 3D Chair. The symbols and labels by each axis identify them throughout this chapter. 


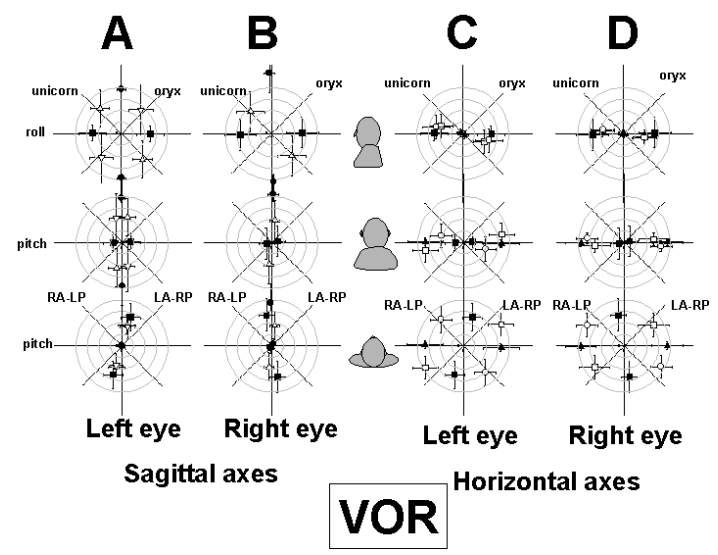

FIGURE 15.2. The orientation and gain of the slow phase of the vestibulo-ocular reflex for each eye elicited by rotation about axes in the sagittal plane $(a, b)$ or horizontal plane $(c, d)$ as seen from the side (top row), back (middle row) and above (bottom row). Each symbol (see Fig. 15.1 for key) represents the tip of a vector obtained by fitting a sine wave to each of the roll, pitch and yaw components of the desaccaded slow phase of the response. The distance from the centre represents the magnitude of the response normalized to the stimulus speed. The circles are at 0.1 intervals. Also shown are the standard deviations in each dimension.

\subsubsection{VOR evoked by rotation about axes in the horizontal plane}

The arrangement of the slow-phase velocity of the VOR evoked by rotation around axes lying in the horizontal plane (roll, LARP, pitch, RALP, see Fig. 1) are illustrated in Fig. 2 (right). Sine waves at the stimulus frequency were fitted to each of the three components (yaw, pitch and roll) of the slow phase of the response. The amplitude of each best-fit sine wave was then divided by the stimulus velocity and treated as the three-dimensional component of a vector (see CD rom for details).

Rotation around the LARP axis evoked a response that was significantly larger in the left eye than the right eye and for RALP the response was larger in the right eye than the left. These differences were primarily due to differences in the pitch component of the response. When the head rolled counter-clockwise (right ear up), evoking a clockwise slow-phase eye roll component, it also evoked an upward component in the left eye and a downward component the right eye: that is a vertical divergence. When rotation was around the RALP axis, the vertical component was larger in the right eye than in the left thus again producing a vertical divergence. Similarly, when rotation was about the LARP axis, the left eye's vertical component was faster than in the right, also leading to a vertical divergence. 


\subsubsection{VOR evoked by rotation about axes in the sagittal plane}

The response to rotation around the roll, yaw, unicorn and oryx axes in the sagittal plane of the head are shown on the left of Fig. 15.2. The clockwise roll of the eyes evoked by the roll component of the roll, unicorn and oryx rotations, was associated with an upward motion of the left eye and a downward rotation of the right eye (Fig. $15.2 \mathrm{a}$ and b). The evoked vertical component had the effect of shifting the response vectors into a plane tilted by approximately 12 degs.

\subsubsection{Uneven gains theory}

Some deviations of the direction of VOR from its evoking stimuli have been noted previously (Crawford and Vilis, 1991) and have been suggested to arise because the different components of the response are generated by independent roll, pitch and yaw systems with different gains (Crawford and Vilis, 1991; Fetter, Zee, Tweed and Koenig, 1994; Solomon, Straumann and Zee, 1997). The uneven gains theory explains the misalignments as following from the different gains of the response evoked by rotation around the cardinal axes of roll, pitch and yaw. Since pitch has a higher gain than roll (pitch 50\%; roll 30\% in this study), the pitch component will dominate the response to a rotation with both pitch and roll components, pulling the overall response towards the pitch direction (Tweed, Sievering, Misslisch, Fetter, Zee and Koenig, 1994).

However, the uneven gains theory always predicts deviations towards the higher gain axis. In fact, responses to rotation about the unicorn and oryx axes (in between roll and yaw) were accurate (Fig. 2a and b, top row). Thus an uneven gains model based on the amplitude of yaw, roll and pitch components measured separately is not adequate.

\subsubsection{The VOR compensates for rotation and translation of the eyes associated with head rotation}

Interestingly the "extra" components of the response that create the misalignments reported here, for example the mysterious up/down components in response to rotation around axes in the sagittal plane, generally turn out to be appropriate to compensate for the translation of the eyes that necessarily accompanies head rotation. For example, because clockwise roll of the head shifts the left eye up and the right eye down, an asymmetrical pitch movement is appropriate (see also Jauregui-Renaud, Faldon, Gresty and Bronstein, 2001). Because the RALP and LARP rotation axes pass quite close to the left and right eyes respectively (see illustration on the CD rom), rotation about these axes causes the more distant eye (left for LARP and right for RALP) to be translated vertically by a considerably larger amount than the eye that is closer to the axis. Clockwise slow- phase eye movement evoked by LARP stimulation was associated with more upward movement of the left eye than of the right and the clockwise eye movement evoked by RALP stimulation was associated with more downward movement of the right 
eye than the left (see Fig. 2). These puzzling directions are thus potentially useful. Attempting to compensate for translation when the two eyes are translating differently necessarily leads to ocular divergence. If the eyes diverge, they cannot maintain their gaze on an individual target. Thus these findings lead to a similar conclusion as that of Groen, Bos and de Graaf, (1999) who suggested that the goal of the compensatory eye movement system is more to stabilize the eye in space than to minimize retinal slip. A similar conclusion was reached by Harris and Mente, (1996) who found the goal of adaptation of the VOR to be directed not towards minimizing retinal slip but to compensating for the best estimate of the movement of the head in space.

The fact that the gain and spatial characteristics of the roll response are not altered when the roll axis is aligned with gravity (Jauregui-Renaud, Faldon, Clarke, Bronstein and Gresty, 1996; Seidman and Leigh, 1989) and that the translationrelated components reported here are found at low frequencies suggests that they do not result from detecting the translation directly. In fact there is no sensory information available anyway to indicate the translation of the eyes due to head rotation in the dark. Although the evidence that these "extra" components are to compensate for translation is circumstantial, nevertheless, to call the deviations of the VOR for the stimulating axis a "misalignment" might be to malign a possible function. This argument is further developed in Harris, Beykirch and Fetter, (in press). Our data are compatible with the notion that the vestibulo- ocular reflex represents an initial core of a compensation not only for angular rotation but also for the translation components of the eye associated with these rotations. Can such an apparently intelligent system be modelled by a simple three-channel system such as we initially proposed?

\subsection{Modelling the VOR as a simple three-channel system}

We took the VOR evoked in each subject about each axis and fitted the whole set with a model that consisted of three channels. Each channel can be regarded as a vector with a gain. There is no intrinsic reference frame for the VOR - neither the structure of canals, nor the Cartesian co-ordinate system of yaw, roll and pitch have any special claims - and so the orientations of the channels as well as their gains were free parameters optimized by a programme (Sigmaplot 6) that chose values for the orientation and gain of the channels and compared the model's oculomotor performance with the data. The model's output was obtained by projecting each stimulus onto the three channels and then multiplying each by the channel's gain. The response was the vector sum of three channel's activities. This process is shown diagrammatically in Fig. 3. The orientation and gains of the channels were systematically varied and the comparison repeated until a best fit was found.

The orientation and gains of the three channels that best fit the data are shown 


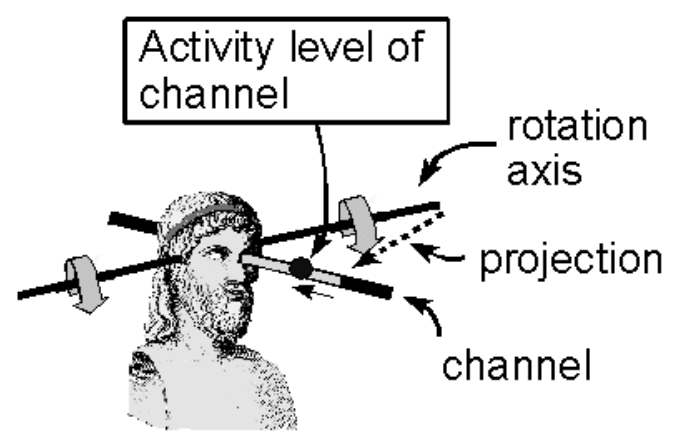

FIGURE 15.3. How the channels were estimated. Three channels (only one shown) were assigned random orientations and gains. The response of each of these hypothetical channels was then calculated by projecting the rotation axis onto the channel (gray bar). The projection is then multiplied by a gain, setting the channel's "activity level", The set of all three "activity levels" are then used as the vector co-ordinates determining the magnitude and direction of the response.

in Fig. 4c. The output of this model in response to rotation around each of the eight axes used in this study are shown in Fig. 4a and d. The model's output (open circles) reproduces all the major features of the pre-adapt data.

\subsubsection{Minimum gain axes}

From the distribution of responses to the tested axes one can extrapolate the direction of the smallest response. This is an interesting direction in our endeavour to model the system with three channels because one channel must align with this "minimum gain axis". If the VOR is generated by the output of three channels, then no response can be less than the vector sum of the three outputs. That is, no one channel can have a gain less than the minimum response. Therefore if one channel has a gain significantly lower than the others (which it does), the location of the minimum response must correspond to when the stimulus is aligned with this channel. Although locating the minimum gain axis was not a method explicitly used to locate the channels, indeed its location (which can be seen from the distribution of gains in the horizontal plane shown in Fig. 5), about 10 degs to the left of straight ahead in the left eye, does correspond to the location of one of the channels abstracted from the overall fit to all the data (see the above view of our proposed channels in the lower panel of Fig. 4c). But it turns out that the minimum gain axis also fits into the other part of our story, the role of the VOR in compensating simultaneously for the translations, as well as the rotation, associated with head movement.

As described above, when the head rotates, it causes both eyes to translate. The only exception is when the axis of rotation of the head passes through the centre of rotation of the eye. Most natural rotations are about the atlanto-occipital joint, 


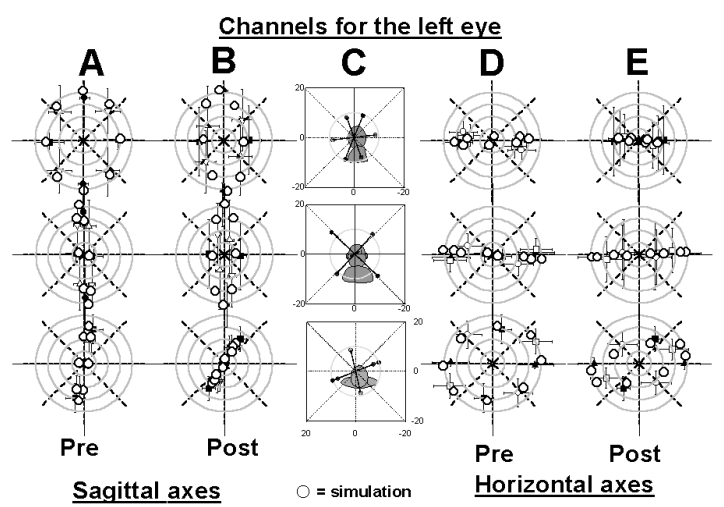

FIGURE 15.4. Simulating the VOR for the left eye. The orientation and relative length of our proposed channels are shown in the centre column (c) from side (top panel), back (middle panel) and above (bottom panel) views. For clarity, the results are divided into those obtained from rotation around axes in the sagittal plane (a, b) and the horizontal plane $(\mathrm{d}, \mathrm{e})$. The average data are shown with standard deviations as symbols (conventions as Fig. 15.2). Superimposed on the data symbols are the output of the model for the preand post-adaptation conditions (large open circles). All the major features of the data are reproduced by the model both before and after adaptation.

which is substantially behind the eyes. Thus the only natural head rotation axis that passes through an eye is around the line joining the atlanto-occipital joint (the approximate centre of head rotation) with the eye's centre of rotation. The minimum gain axis for each eye lines up closely with this line. That is, the minimum gain axis for each eye is closely associated with the only naturally occurring head rotation axis not associated with any translation of that eye.

\subsection{Testing the model}

To test the channel model with the gains and orientations obtained above, we adapted both the subjects and the model and compared the adapted model's predictions with the responses of our adapted subjects.

Our adaptation procedure was to use subject-stationary vision to lower the gain of the vestibulo-ocular reflex around the right-anterior left-posterior axis. Subjects sat in the apparatus and viewed text stuck on the wall of the $1 \mathrm{~m}$ radius sphere. They were instructed to read this text out loud during the adaptation procedure. The sphere on which the letters were attached, moved with the subject thus requiring the VOR to be suppressed. Many experiments have indicated that this procedure results in an adaptive reduction of the gain of the VOR which outlasts the duration of stimulus and persists even when the VOR's performance is measured in darkness (see Berthoz and Melvill Jones, 1985 for a review). Subjects viewed the subject-stationary stimulus during 30 mins of sinusoidal rotation around the 


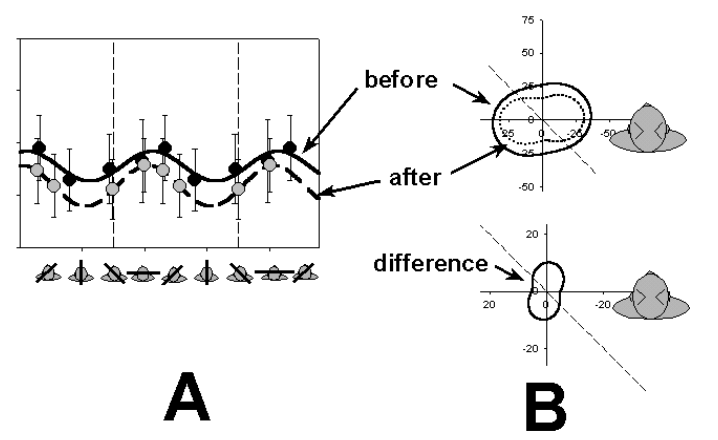

FIGURE 15.5. The effect of reducing the gain around the RA-LP axis. Comparison between before and after responses in the left eye evoked by rotations in the horizontal plane. The data are plotted in linear (a) and polar in above view (b) formats. Sine waves are plotted through the data to visualize the difference between the before and after responses. The difference between these 2 sine waves is plotted in polar co-ordinates in the lower panel of b. The major differences between before and after are around the roll axis.

right-anterior, left-posterior (RALP) axis at a frequency of $0.129 \mathrm{~Hz}$ with a peak velocity of $80 \mathrm{~d} / \mathrm{s}$ and therefore a maximum displacement of \pm 100 degs. Only the left eye's responses were measured after adaptation.

\subsubsection{VOR after adaptation around the RALP axis}

Fig. $4 \mathrm{~b}$ and e shows the pattern of responses for axes in the sagittal and horizontal planes following adaptation. The roll, pitch and yaw components of each of the average responses are also given in table 1 on the $\mathrm{CD}$. The VOR responses evoked by rotation about axes in the sagittal plane normally have a small pitch component which is not required to compensate for such rotations (see section 15.2.3 and Fig. 4b bottom). This pitch component became substantially larger after adaptation. As a result, after adaptation the responses evoked by stimulation axes in the sagittal plane formed a plane tilted by 40 degs (Fig. 4b bottom). The response of the left eye to rotations in the horizontal plane were also substantially altered by our adaptation procedure (compare Fig. $4 \mathrm{~d}$ and e, bottom panels especially). These changes are examined in Fig. 5 which plots a sine wave through the "before" and "after" responses. The difference is largely to be found around the roll axis where the response is much reduced and strongly deviated by the emerging vertical component after adaptation around the RALP axis.

\subsubsection{Adapting the model}

Next the model was "adapted". To do this first the hypothetical activity of each of the channels expected in response to the adapting stimulus was calculated using the gains and orientations of the channels obtained from the best fit to the pre- 
adapted data. For the RALP stimulation used this produced activity in the ratio of $4: 3: 7.5$ in the three channels The gains of each channel were then adjusted by an amount proportional to this activity to produce an "adapted" model (gains going from 0.51: 0.42: 0.34 to 0.33: 0.37: 0.12). The responses of the "adapted" model were simulated by projecting each head rotation onto the "adapted" channels. The output of the adapted model (Fig. 4b and e, open circles) showed an excellent fit to the adapted data, reproducing all the major features of the data described above.

This simple three-channel model thus predicts the normal 3D response to rotation around various axes. Its "deviations" also match the measured deviations in response both before and after adaptation. These "deviations" in turn match the needs of an ocular stabilizing system to deal with the movement of the eyes displaced from the head rotation axis.

\subsection{Significance of the orientations of the channels}

The proposed channels (Fig .4c) do not correspond to canal or Cartesian roll, pitch and yaw co-ordinates. They are far from the planes of individual canals, with one close to roll and the others forming an X approximately in Listing's plane which can be expected to be tilted outwards since the eyes were likely verged in the dark at a distance roughly corresponding to the screen (Mok, Ro, Cadera, Crawford and Vilis, 1992). A separate set of channels is needed for each eye because of this outward twist.

The orientations of the channels that best fit our data, while initially surprising, are in fact compatible with the known neural elements involved in the control of the VOR but which have not been put together in this way before. One channel aligns fairly closely with an axis around which rotation is specially treated by the brain, namely roll movement. Roll movement is processed separately (Crawford, Cadera and Vilis, 1991) and the response to roll rotation has some unique features (Anderson, 1981; Seidman, Paige and Leigh, 1997) suggesting that it is indeed a separate sub-system of the VOR mechanism. The other proposed channels lie roughly in Listing's Plane, again not corresponding to any canal plane. In fact the proposed channels lie close to the intersection of the planes that bisect each canal plane and are thus as close as possible to orthogonal to all of them while having the same distance to each. Positioning the channels thus, optimally uses information from all three canals.

Listing's plane has a central role in the coding of eye movements, especially for saccades (Smith and Crawford, 2001). It has been implicated in the neural substrate of the VOR (Crawford and Vilis, 1991) but here we are making it explicitly the site of two of the VOR's channels or neural co-ordinates. The locations of the channels are thus constrained by Listing's Plane, using information from all canals, being roughly orthogonal to each other, and maintaining body symmetry.

Since the VOR is a phylogenetically ancient eye movement control system, 
found in many animals with no saccadic system (Walls, 1962), the fact that the VOR uses a plane close to Listing's plane, defined in terms of optimal use of information from the canals, may be connected to the evolutionary origin of Listing's plane.

\subsection{The VOR as a post-modern reflex with a simple mechanism}

In summary we can say that the VOR is a post-modern reflex. A central tenant of postmodernism is that nothing can escape its time - everything is context dependent. In this sense, can the concept of a simple, reflexive, input-output vestibuloocular system be maintained? The data presented in this chapter suggest that, despite the sophistication and flexibility of the compensatory oculomotor response to head rotation, the VOR can usefully be regarded as driven by a relatively simple core mechanism. Context specific responses can then be built onto this base to make it a post-modern reflex.

\section{Acknowledgements}

Supported by the Deutsche Forschungsgemeinschaft (MF and KB), the Natural Sciences and Engineering Research Council of Canada (NSERC) (LRH) and the Centre for Research in Earth and Space Technology of Ontario, Canada (CRESTech) (LRH).

\section{References}

Anderson, J. H. (1981). Ocular torsion in the cat after lesions of the Interstitial Nucleus of Cajal. Ann. New York Acad. Sci., 374: 865-871.

Berthoz, A. and Melvill Jones, G. (1985). Adaptive Mechanisms in Gaze Control. Elsevier: New York, NY.

Biguer, B. and Prablanc, C. (1981). Modulation of the vestibulo-ocular reflex in eye-head orientation as a function of target distance in man. In A. F. Fuchs and W. Becker (Eds.) Progress in Oculomotor Research, pp. 525-530. Elsevier North Holland: Amsterdam.

Blum, B. (1991). Channels in the visual nervous system: neurophysiology, psychophysics and models. Freund: London.

Campbell, F. W. and Tegeder, R. W. (1991). A survey of channels and challenges, of information and meaning. In B. Blum (Ed.) Channels in the visual nervous system: neurophysiology, psychophysics and models, pp. 1-10. Freund: London. 
Crawford, J. D., Cadera, W. and Vilis, T. (1991). Generation of Torsional and Vertical Eye Position Signals by the Interstitial Nucleus of Cajal. Science, 252: 1551-1553.

Crawford, J. D. and Vilis, T. (1991). Axes of eye rotation and Listing's Law during rotations of the head. J. Neurophysiol., 65: 407-423.

Curthoys, I. S., Markham, C. H., and Blanks, R. H. I. (1975). The orientation of middle and inner ear structures in cat and man. UCLA Brain Information Service: CA.

Fetter, M., Hain, T. C. and Zee, D. S. (1986). Influence of eye and head position on the vestibulo- ocular reflex. Exp. Brain Res., 64: 208-216.

Fetter, M., Misslisch, H., Sievering, D. and Tweed, D. (1995). Effects of full-field visual input on the three-dimensional properties of the human vestibuloocular reflex. J. Vestib. Res.-Equilib. Orientat., 5: 201-209.

Fetter, M., Zee, D. S., Tweed, D. and Koenig, E. (1994). Head Position-Dependent Adjustment of the 3-Dimensional Human Vestibuloocular Reflex. Acta OtoLaryngologica, 114: 473-478.

Gonshor, A. and Jones, G. M. (1976). Extreme vestibulo-ocular adaptation induced by prolonged optical reversal of vision. J. Physiol. (Lond.), 256: 381414.

Graf, W. and Baker, R. (1983). Adaptive changes of the vestibulo-ocular reflex in flatfish are achieved by reorganization of central nervous pathways. Science, 221: 777-779.

Graf, W. and Baker, R. (1990). Neuronal adaptation accompanying metamorphosis in the flatfish. J. Neurobiol., 21: 1136-1152.

Graf, W. and Simpson, J. I. (1981). The relations between the semicircular canals, the optic axis and the extraocular muscles in lateral-eyed and frontal eyed animals. In A. Fuchs and W. Becker (Eds.) Progress in oculomotor research, pp. 411-420. Elsevier: New York, NY.

Graf, W., Simpson, J. I. and Leonard, C. S. (1988). Spatial-Organization of Visual Messages of the Rabbits Cerebellar Flocculus. 2. Complex and Simple Spike Responses of Purkinje-Cells. J. Neurophysiol., 60: 2091-2121.

Groen, E., Bos, J. E. and de Graaf, B. (1999). Contribution of the otoliths to the human torsional vestibulo-ocular reflex. J. Vestib. Res.-Equilib. Orientat., 9: 27-36.

Harris, L. R. (1997). The coding of self motion. In L. R. Harris and M. Jenkin (Eds.) Computational and Psychophysical Mechanisms of Visual Coding, pp. 157-183. Cambridge University Press: Cambridge.

Harris, L. R., Beykirch, K. and Fetter, M. (in press). The visual consequences of deviations in the orientation of the axis of rotation of the human vestibuloocular reflex. Vis. Res. 
Harris, L. R. and Mente, P. (1996). When vision shifts the vestibulo-ocular reflex, what defines the goal? J. Vestib. Res.-Equilib. Orientat., 6: S91.

Harris, L. R., Zikovitz, D. C. and Kopinska, A. (1998). Frames of reference with examples from driving and auditory localization. In L. R. Harris and M. Jenkin (Ed.) Vision and Action, pp. 66-81. Cambridge University Press: Cambridge, UK.

Hess, B. J. M. and Angelaki, D. E. (1997). Kinematic Principles of Primate Rotational Vestibuloocular Reflex. 2. Gravity-Dependent Modulation of Primary Eye Position. J. Neurophysiol., 78: 2203-2216.

Jauregui-Renaud, K., Faldon, M., Clarke, A., Bronstein, A. M. and Gresty, M. A. (1996). Skew Deviation of the Eyes in Normal Human-Subjects Induced by Semicircular Canal Stimulation. Neurosci. Let., 205: 135-137.

Jauregui-Renaud, K., Faldon, M., Gresty, M. A. and Bronstein, A. M. (2001) Horizontal ocular vergence and the three-dimensional response to wholebody roll. Exp. Brain Res., 136: 79-92.

Leonard, C. S., Simpson, J. I. and Graf, W. (1988). Spatial-Organization of Visual Messages of the Rabbits Cerebellar Flocculus. 1. Typology of Inferior Olive Neurons of the Dorsal Cap of Kooy. J. Neurophysiol., 60: 2073-2090.

Lorente de Nó, R. (1933) Vestibulo-ocular reflex arc. Ann. Neurol. Psychiatry, 30: 245-291.

Malcolm, R. and Jones, G. M. (1970). A quantitative study of vestibular adaptation in humans. Acta Oto-Laryngologica, 70: 126-135.

Misslisch, H. and Hess, B. J. M. (2000). Three-dimensional vestibuloocular reflex of the monkey: optimal retinal image stabilization versus Listing's Law. J. Neurophysiol. 83: 3264-3276.

Misslisch, H., Tweed, D., Fetter, M., Dichgans, J. and Vilis, T. (1997). Interaction of smooth pursuit and vestibulo-ocular reflex in three dimensions. In M. Fetter, T. Haslwanter, H. Misslisch and D. Tweed (Eds.), Threedimensional kinematics of eye, head and limb movements, pp. 191-196. Harwood: Amsterdam.

Mok, D., Ro, A., Cadera, W., Crawford, J. D., and Vilis, T. (1992). Rotation of Listing's Plane during vergence. Vis. Res., 32: 2055-2064.

Müller, J. (1840). Handbuch der Physiologie des Menschen. Vol. II. Holscher: Coblentz.

Oyster, C. W., Takahashi, E. and Collewijn, H. (1972). Direction-selective retinal ganglion cells and control of optokinetic nystagmus in the rabbit. Vis. Res., 12: $183-193$

Paige, G. D., Telford, L., Seidman, S. H. Barnes, G. R. (1998). Human vestibuloocular reflex and its interactions with vision and fixation distance during linear and angular head movement. J. Neurosci., 80: 2391-2404. 
Peng, G. C. Y., Baker, J. F. and Peterson, B. W. (1994) Dynamics of directional plasticity in the human vertical vestibulo-ocular reflex. J. Vest. Res., 4: 453460.

Regan, D. M. (2000). Human Perception of Objects. Sinauer: Sunderland, Mass.

Seidman, S. H. and Leigh, R. J. (1989). The Human Torsional Vestibulo-Ocular Reflex During Rotation About an Earth-Vertical Axis. Brain Res., 504: 264 268

Seidman, S. H., Paige, G. D. and Leigh, R. J. (1997). The VOR during head roll: distinctive properties related to visual demands. In M. Fetter, T. Haslwanter, H. Misslisch and D. Tweed (Eds.) Three-dimensional kinematics of eye, head and limb movements, pp. 171-176. Harwood: Amsterdam.

Shannon, C. E. and Weaver, W. (1949). The Mathematical Theory of Communication. University of Illinois Press: Urbana, IL.

Simpson, J. I., Graf, W. and Leonard, C. (1981). The coordinate system of visual climbing fibers to the flocculus. In A. F. Fuchs and W. Becker (Eds) Progress in Oculomotor Research, pp. 475-484.. Elsevier: North Holland.

Smith, M. A, and Crawford, J. D. (2001). Self-organizing task modules and explicit coordinate systems in a neural network model for 3-D saccades. $J$. Comput. Neurosci., 10: 127-150.

Snyder, L. H. and King, W. M. (1992). Effect of viewing distance and location of the axis of head rotation on the monkey's vestibulo-ocular reflex. I. Eye movement responses. J. Neurophysiol. 67: 861-874.

Solomon, D., Straumann, D. and Zee, D. S. (1997). Three dimensional eye movements during vertical axis rotation: effects of visual suppression, orbital eye position and head position. In M. Fetter, T. Haslwanter, H. Misslisch, and D. Tweed (Eds.) Three-dimensional Kinematics of Eye, Head and Limb Movements, pp. 197-208. Harwood: Amsterdam.

Szentágothai, J. (1950). The elementary vestibulo-ocular reflex arc. J. Neurophysiol., 13: 395-407.

Tweed, D., Sievering, D., Misslisch, H., Fetter, M., Zee, D. and Koenig, E. (1994). Rotational Kinematics of the Human Vestibuloocular Reflex. 1. Gain Matrices. J. Neurophysiol., 72: 2467-2479.

Viirre, E. S. and Demer, J. L. (1995). Effect of Target Proximity on Human Vertical Vestibuloocular Reflex (VOR) During Combined Linear and AngularAcceleration. Invest. Ophthal. and Vis. Sci., 36: S685-S685.

Viirre, E. S., Milner, K., Tweed, D. and Vilis, T. (1986). A Reexamination of the Gain of the Vestibuloocular Reflex. J. Neurophysiol., 56: 439-450.

Walls, G. L. (1962). The evolutionary history of eye movements. Vis. Res. 2: 69-79.

Zeki, S. M. (2001). Localization and globalization in conscious vision. Ann. Rev. Neurosci., 24: 57-86. 